\title{
Evidence for the weak coupling scenario of the Peierls transition in the blue bronze
}

\author{
Bogdan Guster, Miguel Pruneda, and Pablo Ordejón \\ Catalan Institute of Nanoscience and Nanotechnology (ICN2), CSIC and The Barcelona Institute of Science and Technology, \\ Campus Bellaterra, 08193 Barcelona, Spain \\ Enric Canadell \\ Institut de Ciència de Materials de Barcelona (ICMAB-CSIC), Campus Bellaterra, 08193 Barcelona, Spain \\ Jean-Paul Pouget \\ Laboratoire de Physique des Solides, CNRS UMR 8502, Université de Paris-Sud, Universié Paris-Saclay, 91405 Orsay, France
}

(Received 14 January 2019; revised manuscript received 13 March 2019; published 16 May 2019)

\begin{abstract}
On the basis of first-principles DFT calculations the wave-vector and temperature dependencies of the Lindhard response function of the blue bronze $\mathrm{K}_{0.3} \mathrm{MoO}_{3}$ have been calculated. The $k_{F}^{I}+k_{F}^{I I}$ interband component of the response, which is responsible for the Peierls instability, has been quantitatively analyzed. It is found that (i) the electron-hole coherence length of this response determines the length scale of the experimental intrachain CDW correlations, and (ii) the intrachain $q_{\|}$dependence of such a response also determines the shape of the Kohn anomaly experimentally measured. These findings provide compelling evidence that the Peierls transition of the blue bronze $\mathrm{K}_{0.3} \mathrm{MoO}_{3}$ follows the weak electron-phonon coupling scenario in the adiabatic approximation, something that had not yet been proved on the basis of first-principles calculations for a real material. It is proposed that the CDW interchain coupling occurs through a Coulomb coupling between dipolar CDWs. The nature of the phonon mode leading to the dipolar nature of the CDWs is also discussed, and the relevance of these results to rationalize the CDW instabilities in other oxides and bronzes is pointed out. These findings are also contrasted with recent results for other CDW materials like chalcogenides and tellurides.
\end{abstract}

DOI: 10.1103/PhysRevMaterials.3.055001

\section{INTRODUCTION}

Many low-dimensional metallic systems exhibit a charge density wave (CDW) ground state where the electronic density is modulated with a wave number related to the shape of their Fermi surface [1-3]. In experimental situations reported in the literature, the electronic modulation is stabilized by a periodic lattice distortion (PLD) [4]. Thus, electronic and lattice degrees of freedom are coupled by the electron-phonon coupling [5,6]. The electronic energy gain is caused by the fact that the new periodicity of the PLD opens a full gap in the band structure for 1D electronic systems or partial gaps in electronic systems of higher dimension [7]. In the case of 1D systems, the CDW/PLD modulation is accompanied by a metal-insulator transition (i.e., Peierls transition in the literature) and the modulation wave vector, $2 k_{F}$, is simply twice the Fermi wave vector [8].

In the standard description of the Peierls transition [8] the electron-phonon coupling is assumed to be small so that the electronic wave functions are weakly perturbed by the lattice vibrations. In this limit, which relies on a PLD of small amplitude, the instability towards the formation of electronhole pairs is driven by the thermal divergence at a well defined critical wave vector $\left(q_{c}=2 k_{F}\right.$ in the $1 \mathrm{D}$ case $)$ of a sharp electron-hole (i.e., Lindhard [9]) response function of the nonperturbed electron gas. This response can be written as

$$
\chi(q)=-\sum_{i, j} \sum_{k} \frac{f_{F}\left(\epsilon_{i}(k)\right)-f_{F}\left(\epsilon_{j}(k+q)\right)}{\epsilon_{i}(k)-\epsilon_{j}(k+q)},
$$

where $f_{F}$ is the Fermi function and $\epsilon_{i}(k)$ are the band eigenvalues.

In the weak electron-phonon coupling limit and within the adiabatic approximation where $\Omega_{0} \tau_{e h}<1\left(\Omega_{0}\right.$ being the frequency of the bare critical phonon at $q_{c}$ and $\tau_{e h}$ the lifetime of the electron-hole pair), the electron-hole pairs fluctuate so quickly during the phonon oscillation period that they screen the coupling between the atoms. This leads to a phonon softening around $q_{c}$ (i.e., to the occurrence of a Kohn anomaly) for lattice modes whose frozen displacements stabilize the PLD below the Peierls transition. In the adiabatic approximation, the critical lattice dynamics is of the displacive type with a progressive softening of the frequency of the Kohn anomaly when approaching the Peierls transition, following the thermal divergence of the electron-hole response [8]. In this limit the width of the dispersion of the Kohn anomaly is related to the width of the electron-hole response around $q_{c}\left(2 / \xi_{\text {eh }}\right.$, where $\xi_{e h}$ is the coherence length of the electron-hole pair). Finally, in the standard theory of the Peierls transition, the entropy of the critical phonon mode is neglected, an approximation valid if the Kohn anomaly involves only a small fraction of the Brillouin zone (i.e., if $\xi_{e h} 2 k_{F} \gg 1$ in 1D). In the weak coupling limit, the theory of the Peierls transition is of the BCS type [8]. Note that in the presence of a broad electronhole response spreading over a sizable fraction of the Brillouin zone (i.e., if $\xi_{e h} 2 k_{F} \sim 1$ in 1D) the phonon entropy cannot be neglected [10]. In that case it is needed to go beyond the weak coupling BCS formalism to describe the Peierls instability 
and theoretical treatments suggest more likely strong electronphonon coupling scenarios.

In the nonadiabatic approximation $\left(\Omega_{0} \tau_{e h}>1\right)$ the critical $q_{c}$ phonon fluctuates very quickly during the lifetime of the electron-hole pairs so that phonon modes cannot efficiently couple with the electron-hole condensate. There is no screening and thus no critical softening of a phonon branch. The long-living electron-hole condensate induces, via the electronphonon coupling, a quasistatic local PLD quite well decoupled from the phonon spectrum. The critical lattice dynamics of the Peierls transition is thus of relaxation or order-disorder type [11]. Note that such dynamics is also found in the strong electron-phonon coupling limit [12] where the PLD fluctuations are of large amplitude.

The largest contribution of the Lindhard function [Eq. (1)] originates from electronic energies leading to a vanishing denominator. This occurs for electronic states located at the Fermi level such that

$$
E_{F}(k+q)=E_{F}(k) .
$$

Equation (2) defines $k$ states of the Fermi surface (FS) which coincide with other states of the FS after application of a $q$ translation. If there are large portions of the FS exhibiting such a property the nesting of the FS by $q$ will dominantly contribute to $\chi(q)$ [7]. Such a nesting process can simply account for the maxima of $\chi(q)$ and provide a simple explanation for the occurrence of charge and spin density wave instabilities found in many experimental systems [13]. For example, in 1D systems where the FS is made of two planes at the $+k_{F}$ and $-k_{F}$ wave vectors, the translation wave vectors $q= \pm 2 k_{F}$ nest completely the FS leading to a divergence of $\chi(q)$ and to the stabilization of a CDW-Peierls ground state [8]. However, at finite $T$, because of the Fermi-Dirac distribution spreading over several $k_{B} T$ on each side of $E_{F}$, the contributions to the Lindhard function are not restricted to the Fermi level. This is the case for a single band dispersion where the FS nesting wave vector connecting also electronic states with opposite Fermi velocities preserves the divergence.

However, for a more complex band structure with different Fermi velocities, FS nesting is not a sufficient condition to obtain a maximum of $\chi(q)$. We illustrate this statement using a simple model for a 1D metal with two conduction bands having the Fermi wave vector $\pm k_{F}^{I}$ and $\pm k_{F}^{I I}$. If the dispersion of the two bands is parallel, the nesting process linking band dispersions with opposite Fermi velocities leads to maxima of $\chi(q)$ for $q=2 k_{F}^{I}, 2 k_{F}^{I I}$, and $k_{F}^{I}+k_{F}^{I I}$ but not for $k_{F}^{I}-k_{F}^{I I}$ [Fig. 1(a)]. This situation is relevant for the blue bronze considered below [14]. If the dispersion of the two bands is inverted, the nesting process linking band dispersions with opposite Fermi velocities leads to maxima of $\chi(q)$ for $q=2 k_{F}^{I}, 2 k_{F}^{I I}$, and $k_{F}^{I}-k_{F}^{I I}$ but not for $k_{F}^{I}+k_{F}^{I I}$ [Fig. 1(b)]. This situation is relevant for the charge transfer salts of the TTF-TCNQ family [15]. In conclusion, the sign of the Fermi velocity of the different bands connected by the nesting process determines the type of interband contribution leading to maxima of the Lindhard function. With more complex band structures it has been also reported that maxima of $\chi(q)$ could differ from the best FS nesting condition [16]. It is thus necessary to go beyond the simple consideration of the nesting properties of the FS and to perform the direct calculation

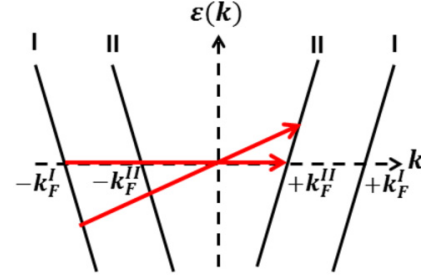

Inter - band $k_{F}^{I}+k_{F}^{I I}$ nesting

(a)

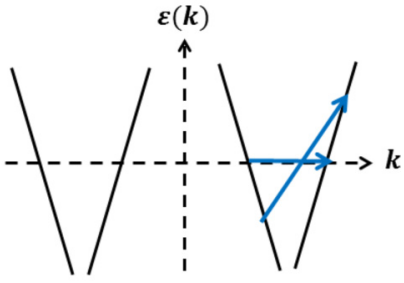

Inter - band $k_{F}^{I}-k_{F}^{I I}$ nesting

(b)
FIG. 1. Schematic illustration of why for a 1D system with two partially filled bands with different Fermi velocities only certain interband nestings may be effective.

of the Lindhard function to obtain the critical wave vectors of the CDW instability. In the case of the blue bronze, the CDW-Peierls instability has been proposed to be caused by the interband $k_{F}^{I}+k_{F}^{I I}$ nesting process [14,17].

The Mo blue bronze $\mathrm{A}_{0.3} \mathrm{MoO}_{3}$, where $\mathrm{A}=\mathrm{K}, \mathrm{Rb}$, or $\mathrm{Tl}$ is a monovalent atom, exhibits a complex C-type centered monoclinic structure [18]. Figure 2(a) shows that this structure is built from $(b, a+2 c)$ layers of $\mathrm{MoO}_{6}$ octahedra. In the perpendicular interlayer $2 a^{*}-c^{*}$ direction vacancies incorporate an ordered sublattice of $\mathrm{A}$ atoms. The essential building blocks of the layers are the clusters of ten $\mathrm{MoO}_{6}$

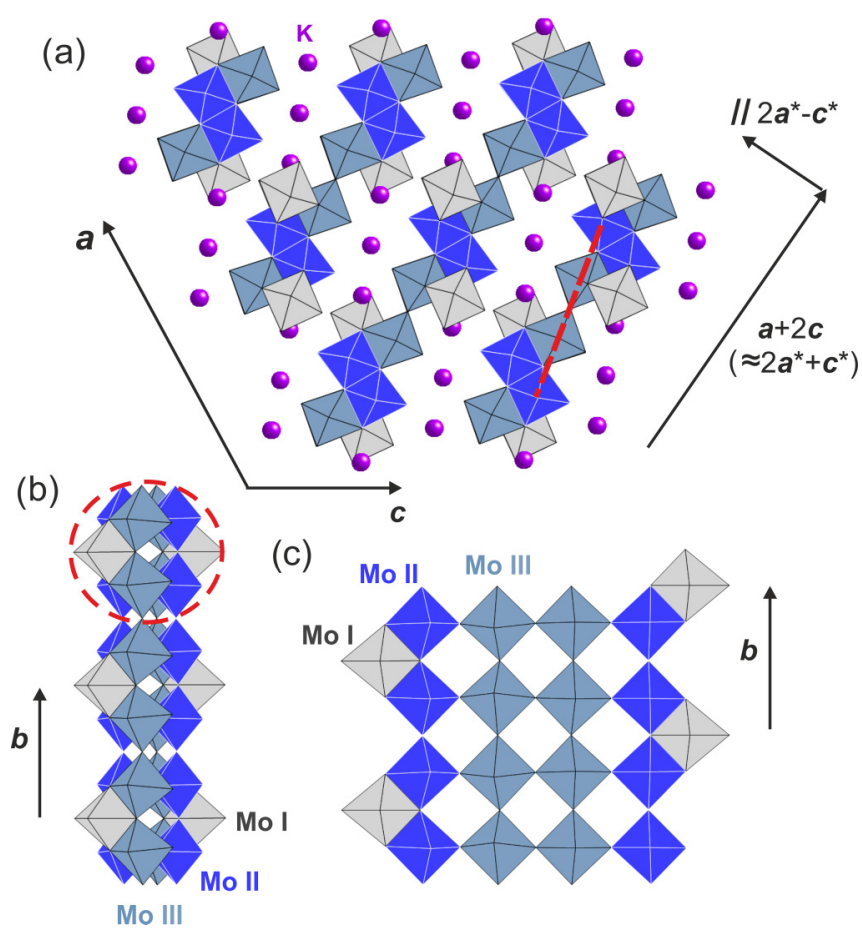

FIG. 2. Crystal structure of the blue bronzes. The lattice vectors shown in (a) are those of the $C$-centered cell of Graham and Wadsley [18]. The three different types of $\mathrm{MoO}_{6}$ octahedra are shown with different colors. The elementary building block of the structure (i.e., a cluster of ten octahedra) and the octahedral chains it generates along the $b$ direction are shown in (b). An alternative description of the octahedral chains associated with the red dashed line in (a) and the hump octahedra is shown in (c). 
octahedra highlighted in Fig. 2(b) (see Sec. III for a detailed structural discussion). Both tight binding [14] and density functional theory (DFT) [19] electronic structure calculations as well as ARPES measurements [20] show that the blue bronze exhibits a quasi-1D band structure with two parallel conduction bands leading to a slightly warped double sheet open Fermi surface. The repeat unit of the crystal structure in the 1D direction is the cluster of ten octahedra. As monovalent A atoms provide three electrons per cluster, the two bands are partially filled with $\rho=3$ electrons. The blue bronze is a 1D metal with a sizable RT conductivity of $\sigma_{b} \sim 10^{3} \mathrm{~S} / \mathrm{cm}$ and an anisotropy of conductivity [21] which follows the structural anisotropy $\sigma_{b}: \sigma_{a+2 c}: \sigma_{2 a *-c *} \sim 1: 10^{-2}: 10^{-3}$. Polarized reflectance measurements show that the blue bronze is a true 1D metal exhibiting a metallic Drude behavior only if the light is polarized in the chain direction [22]. At $T_{p}=180 \mathrm{~K}$ the blue bronze exhibits a Peierls metal-insulator transition accompanied by the setting of a CDW/PLD modulation of critical wave vector $q_{c}=\left(0,1-\left(k_{F}^{I}+k_{F}^{I I}\right), 0.5\right)$ or equivalently, within a reciprocal wave vector, of $q_{c}=\left(1, k_{F}^{I}+k_{F}^{I I}, 0.5\right)$. At low temperature, the value of $k_{F}^{I}+k_{F}^{I I}$ is $\approx 0.75$ [21]. It exhibits a very sizable regime of quasi-1D CDW fluctuations [17] above $T_{p}$ and the occurrence of a Kohn anomaly in the phonon spectrum whose frequency continuously softens as $T_{p}$ is approached [23,24].

In this work we confirm the interband $k_{F}^{I}+k_{F}^{I I}$ nesting mechanism by a direct calculation of the Lindhard function using the real DFT band structure for the undistorted, hightemperature metallic phase of $\mathrm{K}_{0.3} \mathrm{MoO}_{3}$. Our calculation of the thermal dependence of the shape and width of the peaks of the Lindhard function for the metallic phase allows us to quantitatively account for the standard weak coupling scenario of the Peierls transition. Our results do not provide direct insight on the CDW phase, such as the lattice distortions or the specific displacements of the phonon mode exhibiting the Kohn anomaly, but on the driving mechanism for the Peierls transition originating from the instability of the electron gas, and the physical regime of weak electron-phonon coupling and adiabatic limit. To the best of our knowledge, this type of validation of the weak coupling scenario based on actual data for a real material has never been reported in the literature.

\section{COMPUTATIONAL DETAILS}

DFT calculations [25,26] were carried out using a numerical atomic orbitals approach, which was developed for efficient calculations in large systems and implemented in the SIESTA code [27,28]. We have used the generalized gradient approximation (GGA) to DFT and, in particular, the functional of Perdew, Burke, and Ernzerhof [29]. Only the valence electrons are considered in the calculation, with the core being replaced by norm-conserving scalar relativistic pseudopotentials [30] factorized in the Kleinman-Bylander form [31]. The nonlinear core-valence exchange-correlation scheme [32] was used for all elements. We have used a splitvalence double- $\zeta$ basis set including polarization functions [33]. The energy cutoff of the real space integration mesh was 350 Ry. To build the charge density, the Brillouin zone (BZ) was sampled with the Monkhorst-Pack scheme [34] using grids of $(21 \times 45 \times 21) k$ points. The Lindhard response function [Eq. (1)] was obtained from the computed DFT values of the band eigenvalues $\epsilon_{i}(k)$. The integral over $k$ points of the BZ was approximated by a direct summation over a dense, regular grid of points. As the Lindhard function is more sensitive to the accuracy of the BZ integration than the total energy, especially in very anisotropic systems, and/or in the presence of hot spots in the band structure (e.g., saddle points with the corresponding van Hove singularity in the DOS), the $k$-points grid used for its calculation must be more dense than in the standard self-consistent determination of the charge density and Kohn-Sham energy. The calculations are done, nevertheless, using the eigenvalues obtained in the DFT calculation for the coarser grid, and interpolating their values in the denser grid, using a post-processing utility available within the SIESTA package. In this work, for the calculation of the Lindhard response function, the BZ was sampled using a grid of $(256 \times 256 \times 16) k$ points. The two partially filled bands were those taken into account in the calculations.

\section{ELECTRONIC STRUCTURE OF $\mathrm{K}_{0.3} \mathrm{MOO}_{3}$}

$\mathrm{K}_{0.3} \mathrm{MoO}_{3}$ crystallizes in a centered monoclinic structure and contains twenty formula units per unit cell, i.e., $\mathrm{K}_{6} \mathrm{Mo}_{20} \mathrm{O}_{60}$ (Fig. 2) $[18,35]$. In our DFT calculations we used a half-sized unit cell based on the centrosymmetric nature of the crystal where the lattice parameters used are $a^{\prime}=1 / 2(a+b)+c, b^{\prime}=b$, and $c^{\prime}=c$ where $a, b$, and $c$ are the lattice constants reported by Graham and Wadsley [18]. The crystal structure consists of slabs of $\mathrm{MoO}_{6}$ octahedra with the alkali metal atoms in between these slabs. As mentioned, the structure contains three inequivalent Mo atoms and thus three different types of $\mathrm{MoO}_{6}$ octahedra (see Fig. 2). Although the structure of the blue bronzes is usually discussed on the basis of the clusters of ten octahedra shown in Fig. 2(b), an alternative description is more convenient in order to understand the nature of the electronic structure [36] [Fig. 2(c)]. The Mo II and Mo III type octahedra form units of four corner-shared octahedra [marked with a dashed red segment in Fig. 2(a)] which lead to quadruple chains along the $b$ axis by further corner sharing along this direction. The Mo I hump octahedra fill one every two outer holes of these quadrupole chains in an zigzag way sharing two edges with Mo II octahedra [Fig. 2(c)]. Consequently, the chains along $b$ are really built from a repeat unit of ten octahedra: two rows of the four octahedral units and two type I hump octahedra. The cluster of ten Mo octahedra which is the repeat unit of this chain is structurally equivalent to that highlighted in Fig. 2(b). The layers of the blue bronze result from the condensation of these chains along the $a+2 c$ direction through edge sharing of octahedra of the different chains.

The calculated band structure of $\mathrm{K}_{0.3} \mathrm{MoO}_{3}$ along the three main directions of the Brillouin zone is shown in Fig. 3(a). The Fermi level cuts two bands dispersive along $\Gamma$ - $Y^{\prime}$ (i.e., not far from the intrachain $b$ direction, $\Gamma-Y$ ) but only slightly dispersive along $\Gamma-\mathrm{X}^{\prime}$ (i.e., the interchain direction) and with a nil dispersion along $\Gamma-Z^{\prime}$ (i.e., the interlayer direction). The band structure of Fig. 3(a) is in very good agreement with the results of previous DFT calculations [19,37] and different ARPES studies [20,38-45]. For instance, the calculated values of the $k_{F}^{I}$ and $k_{F}^{I I}$ Fermi wave vectors are found to be $0.59 \pi / b$ 


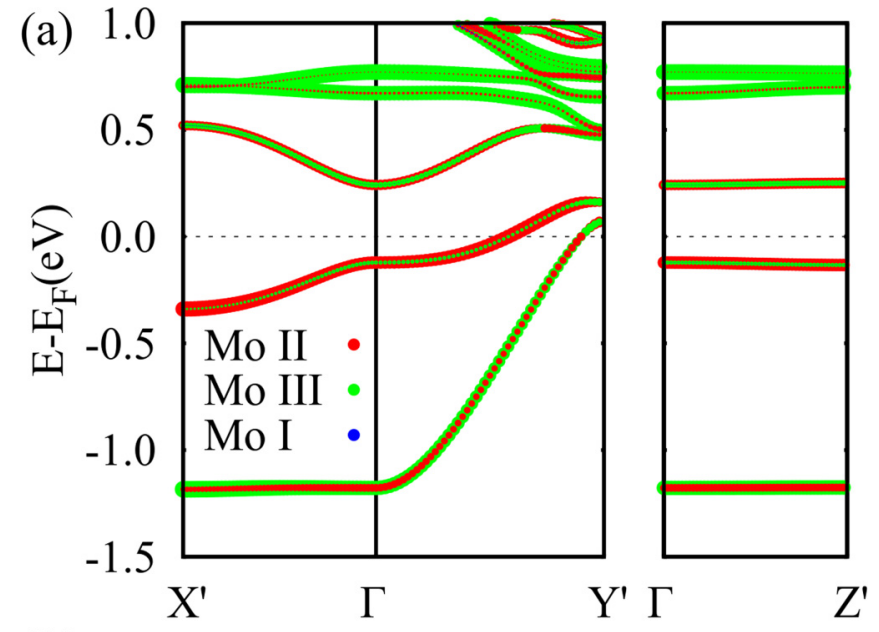

(b)

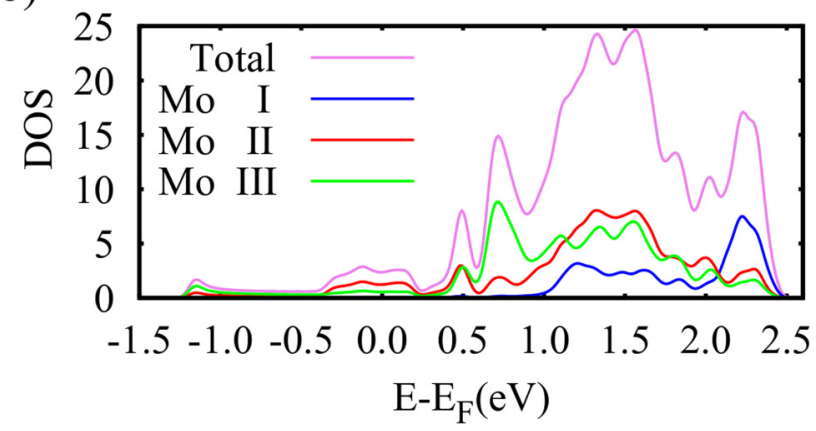

FIG. 3. DFT band structure (a) and density of states (DOS) (b) for $\mathrm{K}_{0.3} \mathrm{MoO}_{3}$. In (a) the size of the blue, red, and green dots are proportional to the Mo I, Mo II, and Mo III character, respectively. $\Gamma=(0,0,0), \mathrm{X}^{\prime}=(1 / 2,0,0), \mathrm{Y}^{\prime}=(0,1 / 2,0)$, and $\mathrm{Z}^{\prime}=(0,0,1 / 2)$ in units of the $a^{\prime *}, b^{\prime *}$, and $c^{\prime *}$ reciprocal lattice vectors (see beginning of Sec. III and Fig. 4). In (b) the total DOS and Mo I, Mo II, and Mo III projected DOS are shown. The DOS is given in units of states per eV per unit cell of $10 \mathrm{Mo}$ and per spin direction.

and $0.91 \pi / b$, respectively, which are within the range of values determined in different ARPES studies: between 0.55 and 0.69 for $k_{F}^{I}$ and between 0.86 and 0.97 for $k_{F}^{I I}$ [20,38-45]. In addition, the two calculated band dispersions are also in good agreement with the ARPES studies. The ratio of the calculated Fermi velocities of the two bands in the $\Gamma-Y$ direction is $\approx 4.5$ which compares well with the more recent ARPES studies, $\approx 4.6$ [45]. Note also that in the Peierls theory of interband nesting [17] an effective Fermi velocity can be defined as $v_{F}=2 v_{F}^{1} v_{F}^{2} /\left(v_{F}^{1}+v_{F}^{2}\right)$, where $v_{F}^{1(2)}$ is the Fermi velocity of band 1 (2). Using the ARPES results of Ref. [45] one obtains $v_{F} \approx 1.9 \mathrm{eV} \AA$, which is in nice agreement with the $v_{F} \approx 2 \mathrm{eV} \AA$ value that we calculate from the slope of the thermal dependence of the electron-hole inverse coherence length (see Fig. 7). Thus, in contrast with earlier tight-binding calculations [14], which led to a pair of bands with similar and considerably smaller dispersion, DFT provides a sound picture of the electronic structure of the blue bronzes. Also shown in Fig. 3(a) is the contribution of the three different Mo atoms to the different bands. Mo I does not contribute to the two partially filled bands. In fact the orbitals of Mo I only participate in levels between 1.0 and $2.5 \mathrm{eV}$ higher than the Fermi level [see Fig. 3(b)]. The reason is that the Mo I octahedra exhibit two strong O-Mo...O short/long bond alternations in the basal plane as a consequence of the fact that half of the octahedron shares edges with Mo II and Mo III octahedra whereas the other half does not share any edge, thus leading to a strong distortion. This structural feature leads to a strong destabilization of the three $t_{2 g}$ orbitals of the Mo I atom [36]. Consequently, the carriers associated with the two partially filled bands of $\mathrm{K}_{0.3} \mathrm{MoO}_{3}$ are confined within the quadruple chains of Mo II and Mo III octahedra. This is in agreement with the fact that the participation of the Mo I atoms in the structural modulation of the blue bronzes was found to be practically nil in the x-ray study of Schutte and De Boer [46]. It is, however, remarkable that, as discussed in detail by Machado-Charry et al. [47], using DFT calculations very similar to the present ones, the brightest spots of the scanning tunneling microscope images of the modulated blue bronzes originate from oxygen atoms of these Mo I octahedra. These atoms are the uppermost oxygen atoms of the surface and the STM experiment is mostly measuring the differences in the local density of states of these $\mathrm{O}$ atoms as a result of the CDW.

The two partially filled bands result from the mixing of the Mo II and Mo III orbitals (as well as the associated O $p$ orbitals), although Mo III dominates in the lower band whereas Mo II dominates in the upper one. These bands are almost exclusively built from the Mo $d_{x z}$ orbitals [here we assume a local system of axis with $z$ along the chain $b$ direction and $x$ along the dashed red line in Fig. 2(a)]. This feature originates from the local distortions of the Mo II and Mo III octahedra, which lead to one strong O-Mo...O alternation in the basal plane of the octahedra along the interlayer direction. Such alternation strongly destabilizes the Mo $d_{y z}$ and $d_{x^{2}-y^{2}}$ orbitals but does not noticeably affect the $d_{x z}$ orbital. The reason is that the Mo $d_{y z}$ and $d_{x^{2}-y^{2}}$ orbitals make $\pi$-type antibonding interactions with the $\mathrm{O} p_{z}$ and $p_{y}$ orbitals, respectively, and the destabilizing effect due to the short Mo-O distance largely outweighs the stabilizing effect of the long Mo-O distance [36]. The inner Mo III octahedra dominate in the lower band because the octahedral distortion is weaker than in Mo II octahedra. The $d_{x z}$ orbitals of the more distorted Mo II octahedra dominate in the upper partially filled band. $\mathrm{K}_{0.3} \mathrm{MoO}_{3}$ is thus a pseudo-1D metal because the $d_{x z}$ orbitals of the Mo II and Mo III quadruple units undergo strong antibonding $\pi$-type interactions with the $\mathrm{O} p_{x}$ orbitals along the chain direction but only weak interchain interactions as a result of the unfavorable orientation of the $d_{x z}$ in different chains.

The calculated Fermi surface for $\mathrm{K}_{0.3} \mathrm{MoO}_{3}$ is reported in Fig. 4. It contains two pairs of slightly warped sheets perpendicular to the $b$ quadruple chains direction. The red sheets, originating from the Mo II octahedra, are found to be slightly more warped. The computed Fermi surface compares very well with those determined by ARPES $[42,45]$ which exhibit a weak but definite warping. In fact, as will be shown in the next section, the warping is practically irrelevant around the temperature of the metal to insulator transition, $T_{p}=180 \mathrm{~K}$ [21], so that the four sheets may be nested by a single interband nesting vector $q$. The calculated Lindhard response function associated with the two partially filled bands is shown in Fig. 5 


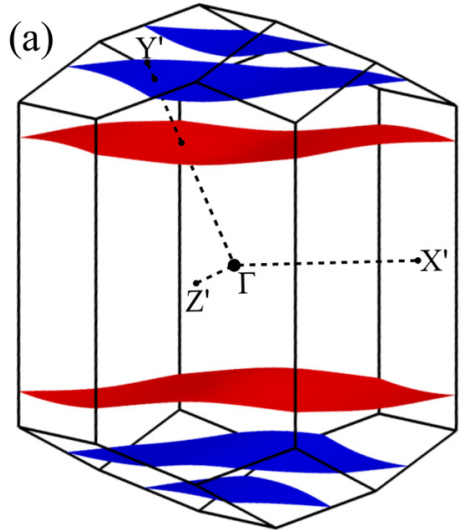

(b)

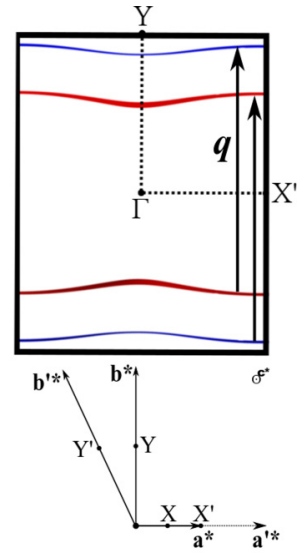

FIG. 4. DFT Fermi surface for $\mathrm{K}_{0.3} \mathrm{MoO}_{3}$ : (a) Representation using the rhombohedral Brillouin zone and (b) view along the perpendicular to the $\left(a^{*}, b^{*}\right)$ plane. In (b) $q$ is the interband nesting vector.

where a clear cusp occurs for $q=0.25 b^{*}$ (note that the chain direction is along $\Gamma-Y$, the green dashed line in Fig. 5). Two weaker maxima are also visible along the $\Gamma-Y$ line. In the next sections we discuss in detail the significance of these results to understand the workings of the CDW/PLD modulation at the origin of the metal to insulator transition of $\mathrm{K}_{0.3} \mathrm{MoO}_{3}$.

\section{QUANTITATIVE ANALYSIS OF THE LINDHARD FUNCTION}

\section{A. The three individual responses}

The Lindhard function (Fig. 5) is made of three responses corresponding to three different nesting processes between the four sets of open Fermi surfaces. Scans along the $b^{*}$ chain direction (see Fig. 6) show [using the labeling of Fig. 1(a)] three peaks at: (i) $1-2 k_{F}^{I I}=0.09 b^{*}$ corresponding to the intraband nesting of the blue FS, (ii) $1-2 k_{F}^{I}=0.41 b^{*}$ corresponding to the intraband nesting of the red FS, and (iii) $1-\left(k_{F}^{I}+\right.$ $\left.k_{F}^{I I}\right)=0.25 b^{*}$ corresponding to the interband FS nesting. The strongest response is for the interband FS nesting. This nesting process achieves the Peierls transition of the blue bronze. With a single modulation all four FS sheets can be connected and thus a gap opens on the entire FS. The intraband I response is stronger than the intraband II response, which agrees with a lesser degree of warping of the corresponding FS. Upon cooling the intensity of these responses increases but does not diverge at low $T$. Divergence is predicted in the case of nested planar FS [7]. Here, the formation of residual pockets in the nesting process between warped FS stops the divergence.

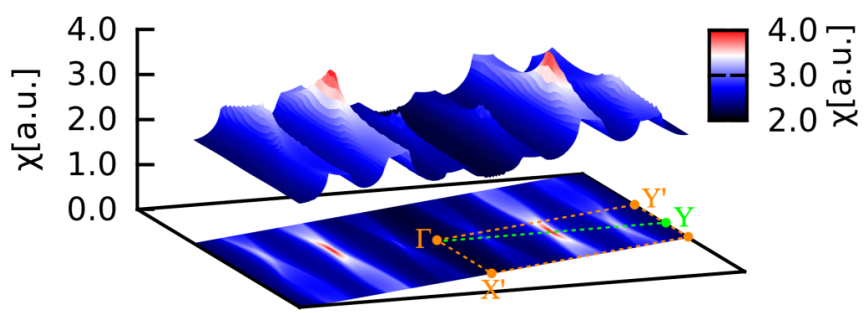

FIG. 5. DFT Lindhard response function for $\mathrm{K}_{03} \mathrm{MoO}_{3}$ at $10 \mathrm{~K}$.

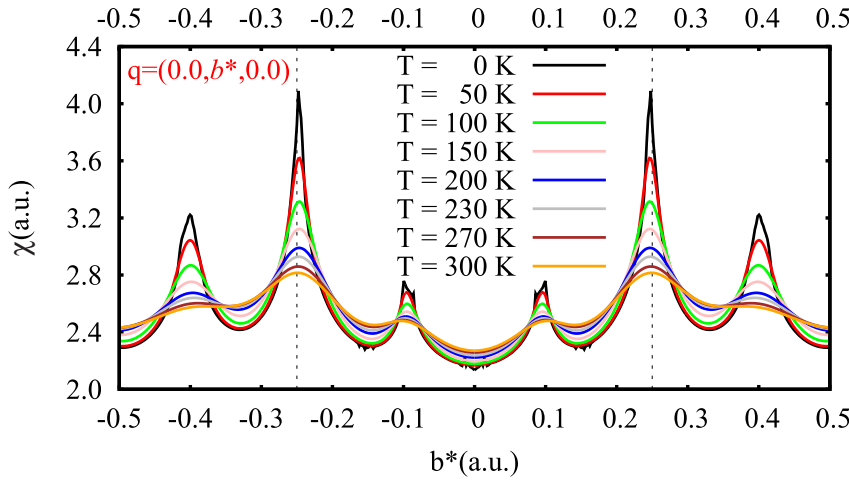

FIG. 6. Scans of the Lindhard response function along the $\Gamma-Y$ chain direction for different temperatures. This figure clearly shows the individual responses of the three Fermi surface nesting processes (i), (ii), and (iii) defined in the text.

Because of the presence of nesting breaking pockets, the three responses exhibit a slightly anisotropic profile at low $T$. The $k_{F}^{I}+k_{F}^{I I}$ interband response exhibits a cusp anomaly whereas a shoulder is exhibited by the $2 k_{F}^{I}$ response and a tilted plateau by the $2 k_{F}^{I I}$ response (see Figs. 5 and 6). The maximum of the interband response occurs for $k_{F}^{I}+k_{F}^{I I}=0.75 b^{*}$, which corresponds to the intrachain component of the experimental modulation wave vector measured at low $T$ [17]. However, the experimental intrachain component decreases significantly by a few percent upon heating [17] while Fig. 6 shows that the maximum of the Lindhard function stays constant at $0.25 b^{*}$ for the whole temperature range considered. Thus, our calculation shows that the experimental decrease of $k_{F}^{I}+k_{F}^{I I}$ is not due to a shift of the $q$ dependence of the Lindhard function induced by thermal population effects of the curved conduction bands, as previously suggested [48]. This point will be further considered at the end of Sec. IV C.

\section{B. Electronic parameters of the interband nesting process and validation of the weak coupling scenario}

Except at very low $T$ when nesting breaking effects are relevant, each individual Lindhard component exhibits a Lorentzian profile in $q$. The half width at half maximum (HWHM) of the Lorentzian in the chain direction gives the inverse coherence length of the fluctuating $1 \mathrm{D}$ electron-hole pair, $1 / \xi_{e h \|}(T)$, which depends on the temperature $T$ and on the microscopic parameters of the 1D electron gas [49]. Figure 7 gives the thermal dependence of $1 / \xi_{e h \|}(T)$ for the interband response component. This quantity increases linearly with the temperature but does not vanish at $0 \mathrm{~K}$, as expected for planar nested FS. The intercept, which amounts to $1 / \xi_{e h \|}^{0}=0.015 \AA^{-1}=1.8 \% b^{*}$, represents the longitudinal size of the pocket due to imperfect longitudinal nesting. One thus has

$$
1 / \xi_{e h \|}(T)=1 / \xi_{e h \|}^{0}+1 / \xi_{e h \|}^{T},
$$

where $1 / \xi_{e h \|}^{T}$ is the thermal length associated with the broadening of the FS with temperature. According to the microscopic theory of Refs. [49,50]

$$
\xi_{e h \|}^{T}=\hbar v_{F} / \pi k_{B} T .
$$




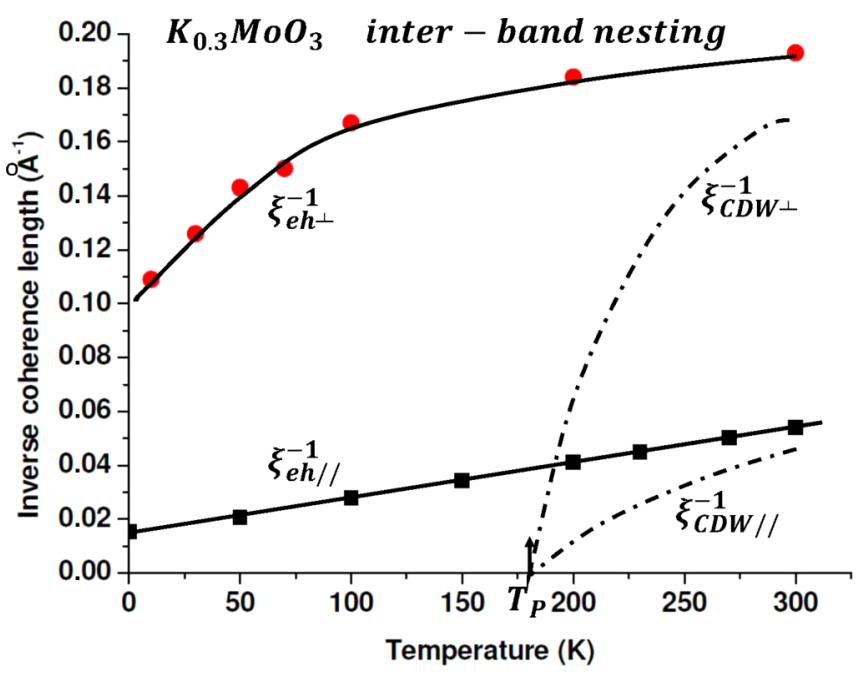

FIG. 7. Thermal dependence of the inverse coherence lengths along the chain $(\|)$ and the intralayer $(\perp)$ directions. These quantities are compared (dashed dotted lines) with the inverse experimental CDW correlation lengths reported in Ref. [51].

For the interband nesting process $v_{F}$ is an effective Fermi velocity defined in Ref. [17] and already quoted in Sec. III. Dividing Eq. (3) by $\left\langle k_{F}\right\rangle=\left(k_{F}^{I}+k_{F}^{I I}\right) / 2\left(=0.31 \AA^{-1}\right)$ leads to the dimensionless expression

$$
1 /\left[\left\langle k_{F}\right\rangle \xi_{e h \|}(T)\right]=1 /\left[\left\langle k_{F}\right\rangle \xi_{e h \|}^{0}\right]+T / T_{\text {eff }}
$$

which, according to the data of Fig 7 , gives a $T_{\text {eff }} \approx 2400 \mathrm{~K}$.

Using the data of Fig. 7 it is found that above $T_{P}$ the value of $\left\langle 2 k_{F}\right\rangle \xi_{e h \|}(T)$ is $\approx 16$ at $T_{P}$ and $\approx 11$ at room temperature (RT). Thus, only a very small fraction of the Brillouin zone is affected by the Peierls critical instability. This justifies the criteria for which the phonon entropy change can be neglected at the Peierls transition. Consequently the weak coupling theory of the Peierls transition should apply to the blue bronzes. Using $T_{\text {eff }}$ the cutoff energy ( $\left.E_{\text {cutoff }}\right)$ entering the standard theory of the Peierls transition can be obtained as $[7,15,50]$

$$
\pi k_{B} T_{\text {eff }}=\hbar v_{F}\left\langle k_{F}\right\rangle=0.65 \mathrm{eV} .
$$

This energy is comparable to the average Fermi energy of the two conduction bands determined by DFT calculation (see Fig. 3) and ARPES measurements [20]. Equation (6) gives an effective Fermi velocity of $v_{F} \approx 2 \mathrm{eV} \AA$.

For a 1D free electron gas with perfect longitudinal $2 k_{F}$ nesting, the Lindhard function should thermally diverge as [50]

$$
\chi\left(2 k_{F}\right)=N\left(E_{F}\right) \ln \left(E_{\text {cutoff }} / \pi k_{B} T\right),
$$

where $N\left(E_{F}\right)$ is the density of states at the Fermi level and where the cutoff energy $E_{\text {cutoff }}$ is of the order of $E_{F}$, as given by Eq. (6). This divergence does not really occur at low $T$ in the blue bronze because of the presence of residual pockets due to the imperfect longitudinal nesting. Under such conditions it is better to use $1 / \xi_{e h \|}(T)$, as given by Eq. (3), instead of the scaling variable $T$. This leads to

$$
\chi\left(2 k_{F}\right) \propto \ln \left[k_{F} \xi_{e h \|}(T)\right]
$$

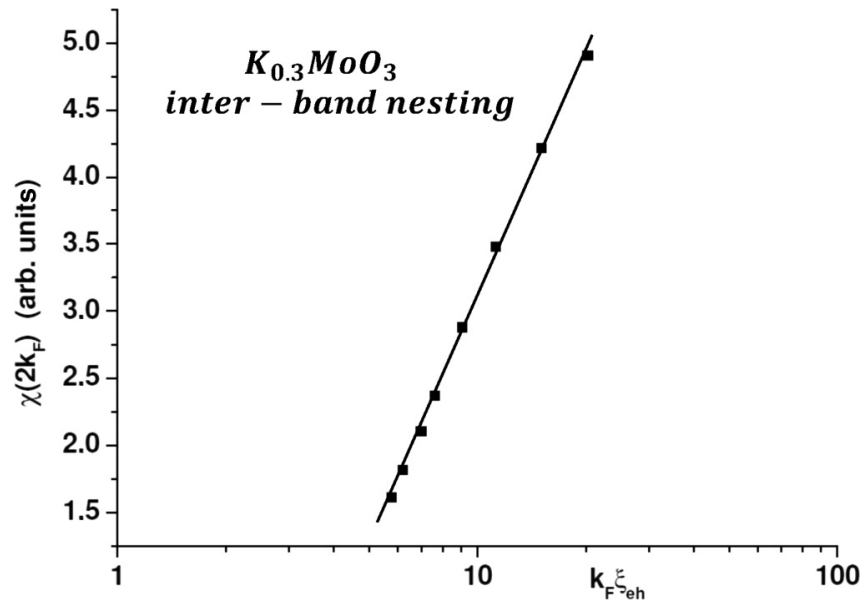

FIG. 8. Logarithmic dependence of the electron-hole response for the interband nesting process of the blue bronze according to Eq. (8).

and, as shown in Fig. 8, this logarithmic dependence is fulfilled by the peak intensity of the interband Lindhard function of the blue bronze for all the temperature range.

The BCS weak coupling relationship relates the Peierls gap at $0 \mathrm{~K}, 2 \Delta_{0}$, with the reduced electron-phonon coupling constant $\lambda[7,15,50]$. For a parabolic band dispersion agreeing with the DFT calculation [see Fig. 3(a)] and ARPES measurements [20] it is found that

$$
\Delta_{0}=4 E_{F} e^{-1 / \lambda}
$$

Then, using the half-optical direct gap in the Peierls ground state [22], $\Delta_{0}=75 \mathrm{meV}$, and $E_{F} \approx 0.65 \mathrm{eV}$ (Eq. 6), a value of $\lambda \approx 0.28$ is obtained. Using the explicit expression for the reduced electron-phonon coupling [7]

$$
\lambda=2 g^{2} N\left(E_{F}\right) / \hbar \Omega_{0},
$$

one can obtain, using the DFT density of states at the Fermi level, $N\left(E_{F}\right)=2.36 \mathrm{eV}^{-1}$ per cluster and spin direction [see Fig. 3(b)], together with a bare critical phonon frequency of $\Omega_{0}=1.7 \mathrm{THz}$ (determined in Fig. 9), $g=20 \mathrm{meV}$. This quantity is more than twice larger than the electron-phonon coupling $g$ determined by the same method for molecular conductors [52].

The finding of $\lambda<1$ and of $\Delta_{0} \ll E_{F}$ sustains the weak coupling approximation for the blue bronze. Figure 7 compares the thermal dependence of the electron-hole coherence length $\left[\xi_{e h \|}(T)\right]$ with the longitudinal CDW structural correlation length $\left[\xi_{\mathrm{CDW} \|}(T)\right]$ measured by X-ray scattering $[17,21,51]$ and whose divergence drives the Peierls transition at $T_{P}$. The CDW/PLD fluctuation divergence is driven by the coupling of the $1 \mathrm{D}$ electron gas with the critical phonon modes via the electron-phonon coupling $g$ [53,54]. More precisely, Fig. 7 shows that at high temperature (i.e., above RT) $\xi_{\mathrm{CDW} \|}(T)$ tends asymptotically towards $\xi_{e h \|}(T)$. Thus, above $\sim 2 T_{P}$, when the coupling to the lattice is not yet critical, the length scale of the CDW fluctuations amounts to the electron-hole coherence length, as expected in the weak coupling scenario of the Peierls transition. 


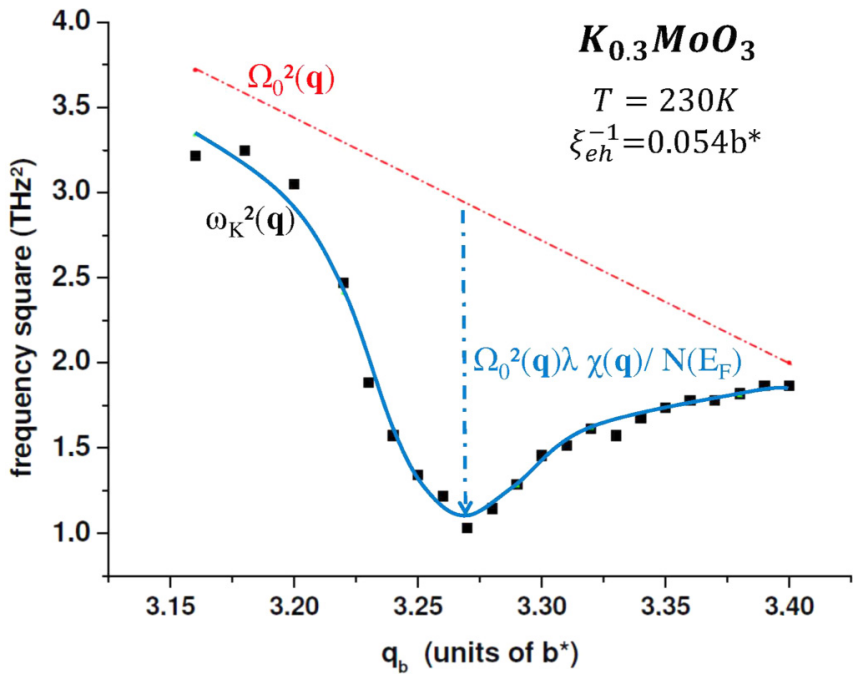

FIG. 9. Fitting of the square of the frequency Kohn anomaly measured at $230 \mathrm{~K}$ in Ref. [24] [see also Fig. 13(a)] with the calculated Lindhard function. The best fit allows us to determine the base line corresponding to the square of the bare critical phonon mode $\Omega_{0}^{2}(q)$.

\section{Relation with the dynamics of the Peierls transition}

Using the relation

$$
\xi_{e h \|}=v_{F} \tau_{e h}
$$

it is possible to obtain the lifetime of the electron pair. It amounts to $\tau_{e h} \approx 10^{-16} \mathrm{~s}$ at room temperature. Thus with $\Omega_{0}=1.7 \mathrm{THz}$, a very small dimensionless quantity $\Omega_{0} \tau_{e h} \approx$ $1.7 \times 10^{-4} \ll 1$ is obtained. This means that the electron-hole pairs fluctuate so quickly during the phonon oscillation period that they are able to screen the coupling constant between the atoms. The Peierls transition of the blue bronze is thus located in the adiabatic limit where the screening effects lead to the formation of a well defined Kohn anomaly in the vicinity of $2 k_{F}$. Such a phonon anomaly is experimentally observed [24]. With the screening effects treated in the RPA approximation the square of the Kohn anomaly frequency is given as [8]

$$
\omega_{K}^{2}(q, T)=\Omega_{0}^{2}(q)\left[1-\lambda \chi(q, T) / N\left(E_{F}\right)\right],
$$

which involves the Lindhard function $\chi(q, T)$. In Eq. (12) we have explicitly included the 3D dependence of the Kohn anomaly [with $q=\left(q_{\|}, q_{\perp}\right)$ ]. Figure 9 shows that Eq. (12) quantitatively accounts for the $q_{\|}$dependence of the Kohn anomaly of the blue bronze in the chain direction at $230 \mathrm{~K}$ using the Lindhard function calculated with DFT. Note that the best fit of the experimental data with the calculated Lindhard function shown in Fig. 9 allows the determination of the square of the bare critical phonon frequency, $\Omega_{0}^{2}(q)$. It is found that $\Omega_{0}\left(k_{F}^{I}+k_{F}^{I I}\right) \approx 1.7 \mathrm{THz}$, which coincides with the frequency of the amplitude mode at low $T$ [24,55].

Note that in the fit of Fig. 9, the $q_{b}$ values of the Lindhard response function have been shifted by about $-0.02 b^{*}$. The reason is the following: Whereas the computed electron-hole response function exhibits a maximum at $0.25 b^{*}$ for the whole temperature range (Fig. 6) because the total number of electrons filling the two conduction bands in the calculation

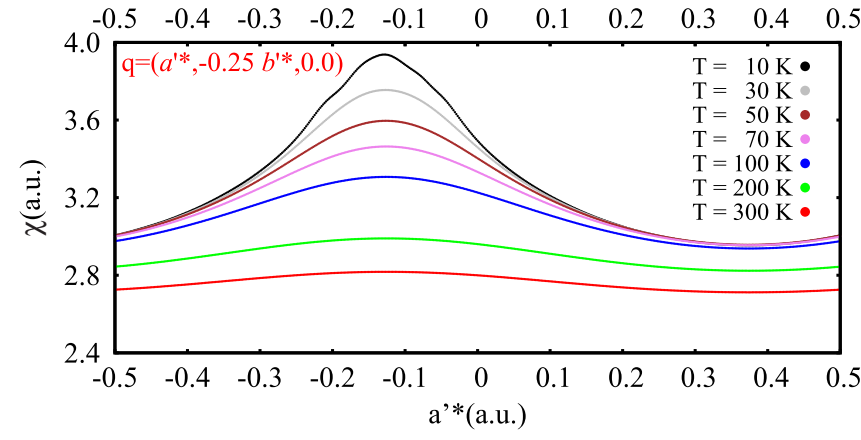

FIG. 10. Scans of the Lindhard response function along the intralayer transverse direction for different temperatures. Note that the maxima at about $0.12 a^{\prime} *$ corresponds to $0 a^{*}$.

remains constant, the wave vector of the experimental minimum frequency of the Kohn anomaly or the $q_{b}$ maximum of the CDW fluctuations [17] continuously increases upon cooling to reach $0.25 b^{*}$ at low temperature. The origin of such a wave vector shift is unclear. Besides the simple yet unlikely explanation that, for some unknown reason, the total number of electrons is changing with temperature, it is possible that the reduced electron phonon coupling $\lambda$ in Eq. (12) depends upon $q_{b}$. According to Eq. (10), $\lambda$ should vary if the bare phonon frequency $\Omega_{0}$ and/or the electron-phonon coupling constant $g$ change with $q_{b}$. The fit in Fig. 9 shows that this is indeed the case for $\Omega_{0}$. The other possibility, a variation of $g$ with $q_{b}$, should also be seriously considered because, as shown by Fig. 13 and discussed in the Appendix, the Kohn anomaly develops in a phonon branch resulting from a strong hybridization between the transverse acoustic $\mathrm{TA}_{2}$ branch and a low frequency optical branch. The two vibration modes certainly involve different atomic displacements so that these lattice deformations should lead to a different electron-phonon coupling. Thus, with a mode mixture changing substantially with $q_{b}$ in the vicinity of $k_{F}^{I}+k_{F}^{I I}$, one should expect that the electron-phonon coupling $g$ should exhibit a strong $q_{b}$ dependence.

\section{Interchain coupling}

In a purely $1 \mathrm{D}$ electron-phonon coupled system the intrachain correlation length diverges at $0 \mathrm{~K}$ because the structural fluctuations prevent any ordering at finite temperature [53,54]. The observation of a Peierls transition at finite $T$ is due to the interchain coupling between the CDW fluctuations [54]. In general interchain coupling can occur through three different mechanisms [11,54]:

(i) a transverse nesting of the FS. In that case the Lindhard function should exhibit an interchain wave-vector dependence (i.e., $q_{\perp}$ ) along which direction the FS is warped,

(ii) an interchain Coulomb coupling between CDW's, since each individual CDW exhibits a charge modulation, or

(iii) a transverse wave-vector, $q_{\perp}$, dependence of the bare critical phonon $\Omega_{0}(q)$.

It is worth considering the likeliness of the three coupling mechanisms for the blue bronze. Let us start with mechanism (i). Figure 10 presents thermal scans of the Lindhard function along the $a+2 c \approx 2 a *+c^{*}$ intralayer transverse direction 


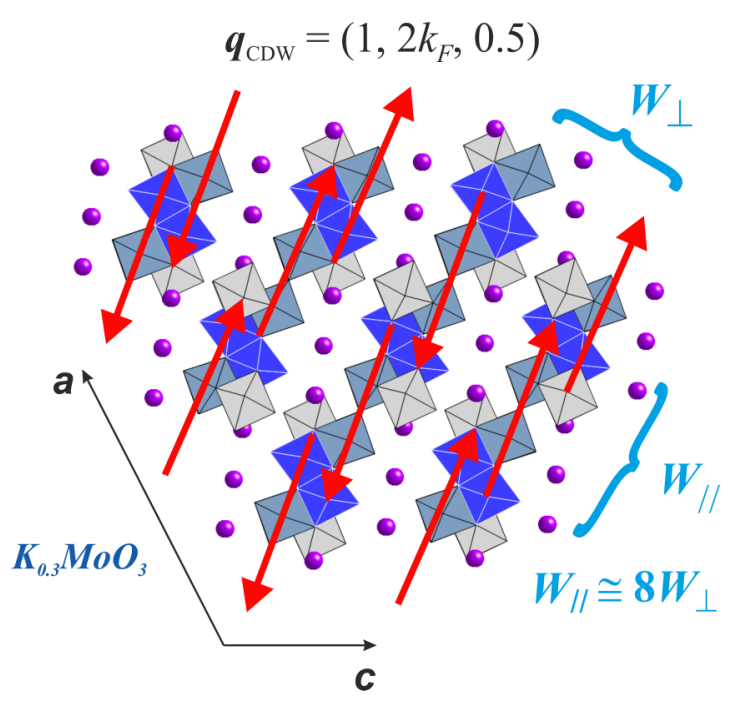

FIG. 11. Lateral phasing of the dipolar CDWs in $\mathrm{K}_{0.3} \mathrm{MoO}_{3}$.

(see Fig. 2). It exhibits a very broad response centered at $q_{\perp}=0$ [in the $a^{*}, b^{*}$ frame defined in Fig. 4(b)] corresponding to an interchain coupling in phase along the $a+2 c$ direction. This wave vector achieves the best FS nesting of band I to band II, as outlined in previous band structure calculations $[14,19]$. Note however that mechanisms (b) and (c), which will be considered below, give a similar phasing. However the present calculation shows that the transverse response of the Lindhard function is quite broad. From its HWHM one gets an inverse interchain electron-hole coherence length $\xi_{\text {eh } \perp}^{-1} \approx 0.19 \AA^{-1}$ at RT which very slightly decreases upon cooling (Fig. 7), reaching $0.18 \AA^{-1}$ at $T_{P}$. The inverse of this quantity leads to a coherence length of $\sim 5.5 \AA$ which is about the distance between the two outer Mo III of the cluster but twice smaller than the distance ( $12 \AA$ ) between the two outer Mo II of the segment defined in Fig. 2(c). $\xi_{\text {eh }}^{-1}$ is larger than the inverse CDW structural correlation length $\xi_{\mathrm{CDW} \perp}^{-1} \sim$ 0.14-0.17 $\AA^{-1}$ measured along the $2 a *+c^{*}$ direction at RT $[17,51]$. In addition, the nondetection of a transverse plasma edge for light polarized in the transverse direction [51] shows that the warping effect of the FS is not thermally relevant above $T_{P}$. The blue bronze thus remains a true $1 \mathrm{D}$ metal in the CDW fluctuating regime above $T_{P}$. We thus conclude that mechanism (i) is not relevant for the blue bronze and we must concentrate on the other two mechanisms.

Let us first consider mechanism (ii) which was previously discussed in Ref. [11]. According to the structural refinement of the modulated structure of the blue bronze below $T_{P}$ [46] there is basically a longitudinal displacement of the Mo atoms in the direction of each linear segment of four corner-sharing $\mathrm{MoO}_{6}$ octahedra [see Fig. 2(c)]. This in-phase displacement induces a ferroelectric polarization of the segment, and the CDW modulates along $b$ the amplitude of polarization of linear segments with the $2 k_{F}=0.75 b^{*}$ wave number. In this scenario, the interchain electrostatic coupling mechanism occurs between dipolar CDW. Such a coupling is quite anisotropic and as shown in Fig. 11:

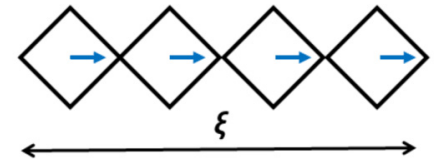

(a)

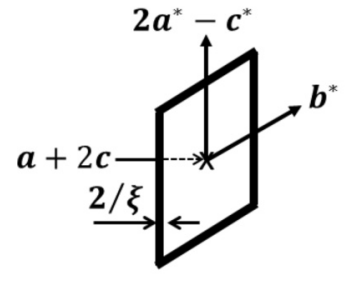

(b)

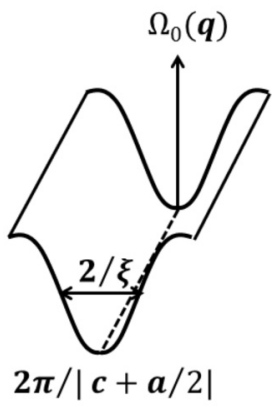

(c)
FIG. 12. Illustration of the low-frequency phonon coupling mechanism for interchain coupling in the blue bronze and its detection. (a) Local polarization induced by the correlated Mo displacements in a segment made of corner sharing octahedra. (b) Planar diffuse scattering developed in x-ray scattering measurements due to the correlated Mo displacements shown in (a). (c) A sheet of low-frequency phonons perpendicular to the polarization direction.

(i) the intralayer coupling along $a+2 c$ between polar CDWs in the dipole direction, $W_{\|}$, leads to a uniform interchain CDW order,

(ii) the interlayer coupling along $2 a^{*}-c^{*}$ between polar layers of CDWs in the perpendicular direction, $W_{\perp}$, leads to a staggered interlayer CDW order.

From the anisotropy of inter- and intralayer transverse correlation lengths [4] one gets an anisotropy of couplings of $\mathrm{W}_{\|} \approx 8 \mathrm{~W}_{\perp}$ (see Fig. 11).

Finally, let us consider mechanism (iii) above $T_{P}$. This mechanism is compatible with mechanism (ii) below $T_{P}$. Mechanism (iii) relies on the presence of an incipient anisotropic valley of low frequency phonons whose bare frequency $\Omega_{0}(q)$ sizably varies with $q$. In the blue bronze such an anisotropic dispersion along $q_{\perp}$ is observed for the acoustic phonon branch polarized along $a+2 c$ mixed with a polar optical branch (see Appendix for experimental evidence taken from the phonon spectrum). This composite mode involves optical-like uniform off-center Mo displacements within the four $\mathrm{MoO}_{6}$ octahedra of each segment. The correlated Mo displacement between neighboring corner-sharing octahedra should induce a local polarization [Fig. 12(a)]. When the correlated Mo displacements are in phase between neighboring overlapping segments of four octahedra separated by $a / 2+c$ [see Fig. 2(a)], a frequency softening of the composite acoustic/optical phonon branch should occur. This softening persists along the $b^{*}$ and $2 a^{*}-c^{*}$ wave vectors perpendicular to the segment direction and starting from each $2 \pi /|a / 2+c|$ reciprocal position. In reciprocal space this should give rise to a sheet of low frequency phonons perpendicular to the polar segment direction [Fig. 12(c)]. The trace of such a sheet of low frequency phonons leads to an enhanced x-ray planar diffuse scattering [Fig. 12(b)] which has been detected in the blue 
bronze at RT (see Refs. [11,21]). The CDW/PLD instability and the associated Kohn anomaly develop inside this valley of soft phonons. Such a preexisting valley imposes the intralayer CDW component $q_{\perp}=0$. We thus suggest that mechanisms (ii) and (iii) which are strongly intermingled are at the origin of the interchain coupling in the blue bronzes.

Similar low frequency planar valleys of mixed acoustic/polar phonons are observed in ferroelectric perovskites such as $\mathrm{BaTiO}_{3}, \mathrm{KNbO}_{3}$, and $\mathrm{KTaO}_{3}$ [56-58] or the chainlike ferroelectric SbSi [59]. Note that features similar to those of the blue bronze are observed in the CDW monophosphate tungsten bronzes, $\left(\mathrm{PO}_{2}\right)_{4}\left(\mathrm{WO}_{3}\right)_{2 m}$, which are built from segments of $m$ corner-sharing $\mathrm{WO}_{6}$ octahedra and which exhibit a tendency to ferroelectricity. Note that $\mathrm{WO}_{3}$, which corresponds to the limit of this family when $m \rightarrow \infty$ is an antiferroelectric. The CDW/PLD instability in the monophosphate tungsten bronzes develops inside a planar valley of low frequency phonons also perpendicular to the segment directions $[60,61]$. The possible link between the CDW/PLD instabilities of the blue bronzes, $\gamma-$ and $\eta-\mathrm{Mo}_{4} \mathrm{O}_{11}$ Magnéli phases and monophosphate tungsten bronzes suggested by our discussion is thus a challenging issue in the quest for a full understanding of microscopic origin of the CDW instabilities in oxides and bronzes.

\section{COMPARISON WITH OTHER CHARGE DENSITY WAVE SYSTEMS}

The present study shows that the CDW instability and the Peierls transition of the blue bronze can be quantitatively understood within the weak electron-phonon coupling scenario. As a consequence, there is a clear softening above $T_{P}$ of a sharp Kohn anomaly driven by the divergence of the electronhole response function (Fig. 9). This well pronounced Kohn anomaly leads to the emergence below $T_{P}$ of two collective excitations of the amplitude and phase of the incommensurate CDW modulation whose dispersion has been clearly measured by inelastic scattering $[24,62,63]$. The phase excitation mode whose frequency tends to zero at the $2 k_{F}$ wave vector and which stiffening of the linear dispersion increases upon cooling [62,63], is a basic ingredient allowing to understand the sliding of the CDW of the blue bronze under electric fields [1-3]. This situation should be contrasted with the case of $\mathrm{NbSe}_{3}$ where analogous CDW sliding effects have been reported [64]. Two differences are worth noting. First, the Lindhard function of the blue bronze shows well decoupled $2 k_{F}$ sharp responses for the different intra- and interband FS nesting processes (Fig. 6) while $\mathrm{NbSe}_{3}$ shows a broad electron-hole response in the chain direction where the different intra- and interband FS nesting processes are superposed [65]. Second, up to now no Kohn anomaly has been detected in $\mathrm{NbSe}_{3}$ by neutron and $\mathrm{x}$-ray inelastic scattering methods [66]. Such an absence of Kohn anomaly has been interpreted as providing evidence for a strong coupling scenario of the Peierls transition [66]. Note however that these measurements report an anomalous drop of the frequency of the longitudinal acoustic (LA) branch for wave vectors tending to the zone boundary. Thus, it is expected that in the next Brillouin zone the LA branch should transform into an longitudinal optical (LO) branch. This LO branch, which has the right symmetry to achieve the out-of-phase longitudinal deformation of first neighbor coupled $\mathrm{NbSe}_{3}$ chains required to form a $\pi$ shifted CDW [67], could exhibit a soft frequency on a large intrachain wave vector range around the CDW critical wave number. In this respect note that, in agreement with this hypothesis, both the Lindhard function calculation [65] and the measurement of the intrachain CDW correlation length [68] give a small $\xi_{\text {eh }} \sim 5-10 \AA$ at RT which leads to a product $2 k_{F} \xi_{\text {eh }} \sim 2-4$, more than three times smaller than $2 k_{F} \xi_{\text {eh }} \approx 11$, estimated here for the blue bronze. This indicates that the phonon entropy should certainly not be neglected when considering the mechanism of the Peierls transition of $\mathrm{NbSe}_{3}$. In this respect, $\mathrm{NbSe}_{3}$ could bear a resemblance to the $2 \mathrm{D} \mathrm{CDW}$ systems which will be considered below.

It is also interesting to compare our findings to those for $2 \mathrm{D}$ CDW systems such as the $\mathrm{RTe}_{3}$ (where $\mathrm{R}$ is a rare earth atom) tellurides and the transition metal dichalcogenides. In $\mathrm{TbTe}_{3}$, the CDW lattice instability is revealed by the formation of a broad Kohn anomaly in an optical branch where frequency softens and damping increases at the critical CDW wave number when approaching the Peierls transition $\left(\mathrm{T}_{P}=330 \mathrm{~K}\right)$ [69]. Furthermore, below $\mathrm{T}_{P}$ the CDW modulation develops with a critical wave vector slightly off the best nesting FS wave vector [16,69]. It is thus proposed [69] that a wave vector dependent electron-phonon coupling could be responsible for this effect.

Controversial interpretations have been proposed during many years concerning the mechanism of the CDW instability of the layered transition metal dichalcogenides. According to a recent review [70], predictions based on the meanfield model agree only semiquantitatively with experimental data and it appears that generally there is no real dominant factor favoring the $\mathrm{CDW}$ formation. A recent inelastic $\mathrm{x}$ ray investigation of $2 \mathrm{H}-\mathrm{NbSe}_{2}$ evidences the formation of a broad and damped Kohn anomaly in a longitudinal acoustic branch whose frequency critically softens at the CDW wave number when reaching $\mathrm{T}_{P}=33 \mathrm{~K}$ (contrary to earlier neutron scattering investigations) with a damping diverging at the CDW transition [71]. As it is the case for $\mathrm{TbTe}_{3}$ [69], it was proposed [71] that in the presence of a broad electron-phonon response, which does not really select a particular FS nesting mechanism [16], the $q$-dependent electron-phonon coupling should be incorporated into the explanation of the CDW instability.

Compared to $\mathrm{NbSe}_{3}$, rare-earth tellurides and transition metal dichalcogenides, the blue bronze, which exhibits a CDW instability that can be accounted for by the standard (weak electron-phonon coupling) Peierls scenario, appears to be unique. This is due to the fact that it exhibits a sharp $2 k_{F}$ electron-hole response, due to a simple FS nesting mechanism, which drives the formation of a sharp Kohn anomaly which softening drives the Peierls/CDW instability. In rare-earth tellurides and transition metal dichalcogenides, the electron-hole response is broad and leads to the formation of a broad Kohn anomaly. In the latter 2D CDW metals the way by which the critical CDW wave vector is selected remains unclear. It has been recently proposed that a wave-vector dependent electron coupling is essential for that purpose [71]. Another important question concerns the mechanism of the CDW/Peierls instability. In the presence 
of a broad Kohn anomaly, where the softening involves a large number of wave vectors of the Brillouin zone, the lattice degrees of freedom play a more important role (in particular through its entropy) than that assigned in the standard theory of the Peierls transition, where only the elastic energy cost is considered. A full account of lattice effects, considered earlier by McMillan [10], should invalidate the BCS-type theory for the Peierls transition and should lead to strong coupling scenarios.

Finally, let us mention that other 2D transition metal Mo and $\mathrm{W}$ metallic oxides and bronzes exhibit a succession of CDW instabilities [1,3]. In contrast with the transition metal dichalcogenides, the electronic structure of these oxides can be described by a 2D lattice of interpenetrating and differently oriented chains [72]. As a consequence, their Lindhard function, which is dominated by the $2 k_{F}$ FS nesting processes of individual chains, exhibits in reciprocal space a collection of differently oriented and well-defined chainlike electronhole responses similar to that calculated for the blue bronze [73,74]. The CDW instability occurs at the crossing point of the differently oriented chain responses, whose associated 1D-like CDW fluctuations have been detected by x-ray diffuse scattering methods [75]. The CDW transition of these materials thus presents a Peierls character [61]. However since inelastic scattering measurements have not been performed, the CDW lattice dynamics is still unknown.

\section{CONCLUDING REMARKS}

The wave-vector and temperature dependencies of the electron-hole (i.e., Lindhard) response function of the blue bronze $\mathrm{K}_{0.3} \mathrm{MoO}_{3}$ have been calculated on the basis of its first-principles DFT electronic structure. This response has three components corresponding to three possible nesting processes of its warped double sheet quasi-1D FS. We have quantitatively analyzed the $k_{F}^{I}+k_{F}^{I I}$ interband electron-hole component which is responsible for the Peierls instability of the blue bronze. We have shown that the electron-hole coherence length of this response determines the length scale of the experimental intrachain CDW correlations. In addition, the intrachain $q_{\|}$dependence of such a response also determines the shape of the Kohn anomaly experimentally measured. All these features prove that the Peierls transition of the blue bronze can be well accounted for by the weak electron-phonon coupling theory in the adiabatic approximation. To the best of our knowledge this is the first time that such a theory, already established in the 1970s, is quantitatively verified. Finally, the calculation of the intralayer transverse $q_{\perp}$ dependence of this response shows that the warping effect of the FS above $T_{P}$ does not provide a pertinent mechanism for the CDW interchain coupling. We propose that such interchain coupling is achieved through the Coulomb coupling between dipolar CDWs. Furthermore, we show that the dipolar nature of the CDW modulation is due to the condensation at $T_{P}$ of a critical phonon mode resulting from the hybridization of an acoustic branch polarized in the $\mathrm{MoO}_{6}$ intralayer segment direction and an optical polar branch similar to those found in ferroelectric perovskites. We suggest that such an mechanisms also occur in CDW oxides and bronzes such as the monophosphate tungsten bronzes.

\section{ACKNOWLEDGMENTS}

This work was supported by Spanish MINECO (the Severo Ochoa Centers of Excellence Program under Grants No. SEV-2017-0706 and No. SEV-2015-0496), Spanish MICIU, AEI and EU FEDER (Grants No. PGC2018-096955-B-C43 and No. PGC2018-096955-B-C44), Generalitat de Catalunya (Grant No. 2017SGR1506 and the CERCA Programme), and the European Union MaX Center of Excellence (EU-H2020 Grant No. 824143).

\section{APPENDIX: CRITICAL LOW FREQUENCY PHONON BRANCHES OF $\mathrm{K}_{0.3} \mathrm{MoO}_{3}$}

The phonon spectrum of $\mathrm{K}_{0.3} \mathrm{MoO}_{3}$ is quite complex because it contains many optical branches of low frequency which hybridize with acoustic branches. Figure 13(a) shows the dispersion of some of these branches measured by inelastic neutron scattering between $225 \mathrm{~K}$ and RT along the reciprocal directions defined in Figs. 13(b) and 13(c) [24,76]. Since previous neutron scattering studies focused on the dynamics of the Kohn anomaly, the phonon spectrum of the blue bronze has never been analyzed. In this respect the true polarization of the critical phonon branch has never been considered. This is the purpose of this Appendix. Let us first consider the acoustic branches. The orthogonal frame used to label them is based on the structural anisotropy [see Fig. 2(a)] according to the decrease in the bonding strength:

(i) the monoclinic $b$ direction: label 1 ,

(ii) the [102] direction: label 2,

(iii) the perpendicular to the $(-201)$ layer of $\mathrm{MoO}_{6}$ octahedra: label 3.

Using the slope of the acoustic branch one obtains the elastic constants given in Table I. The relative $C_{i j}$ values follow the structural anisotropy:

(i) for the compression deformation: $\mathrm{C}_{22}>\mathrm{C}_{11} \gg \mathrm{C}_{33}$, the deformation energy of the chain of clusters is stronger than that between clusters along the [102] direction of the layer and much stronger than that in the interlayer direction incorporating alkaline atoms.

TABLE I. Elastic constants obtained from the dispersion of the acoustic branches shown in Fig. 13(a). The direction of propagation $q$ and the polarization $e$ of the acoustic branch used for each determination are indicated. The accuracy of the determination is estimated to be of 5\%. A comparison between our $\mathrm{C}_{i j}$ values and other measurements in the literature can be found in Ref. [77].

\begin{tabular}{lccc}
\hline \hline Elastic constant & $q$ direction & $e$ direction & $\mathrm{C}_{i j}\left(10^{2} \mathrm{GPa}\right)$ \\
\hline $\mathrm{C}_{11}$ & 2 & 2 & 1.5 \\
$\mathrm{C}_{22}$ & 1 & 1 & 2.25 \\
$\mathrm{C}_{33}$ & 3 & 3 & 0.31 \\
$\mathrm{C}_{44}$ & $3 / 1$ & $1 / 3$ & 0.21 \\
$\mathrm{C}_{55}$ & 2 & 3 & 0.22 \\
$\mathrm{C}_{66}$ & $1 / 2$ & $2 / 1$ & 0.52 \\
\hline \hline
\end{tabular}




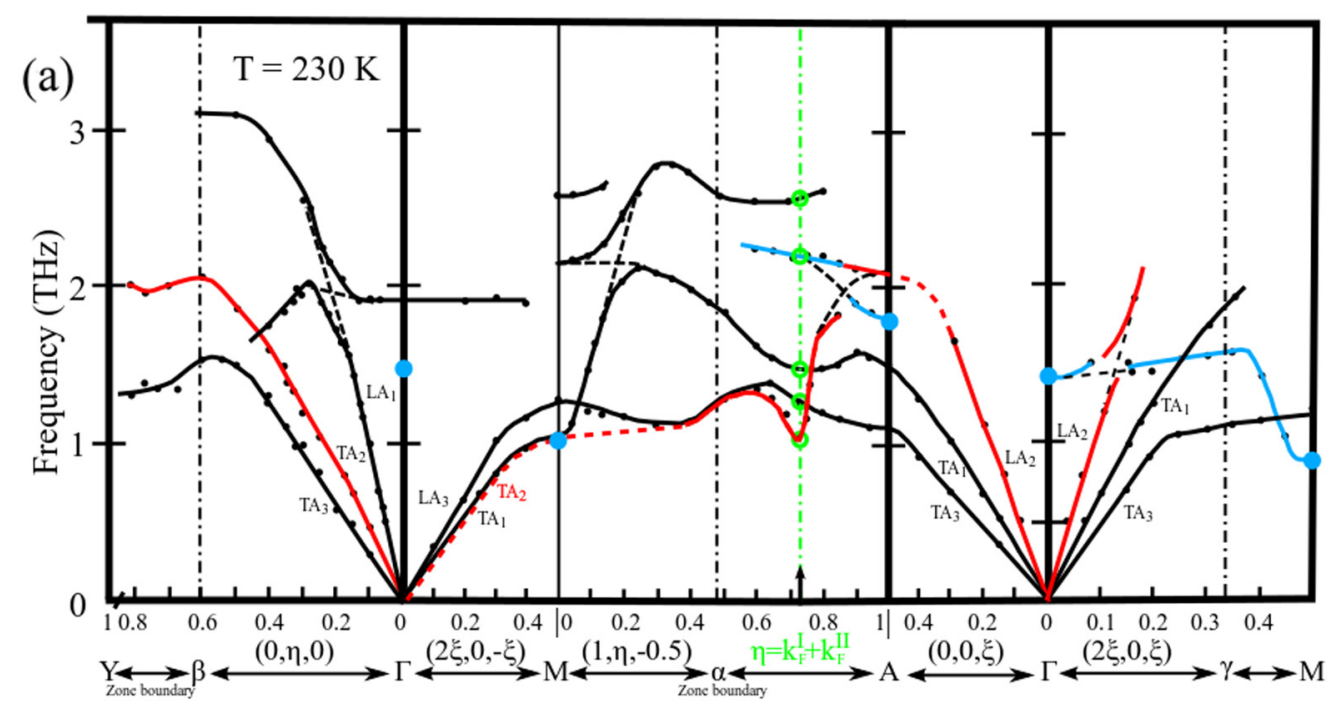

(b)

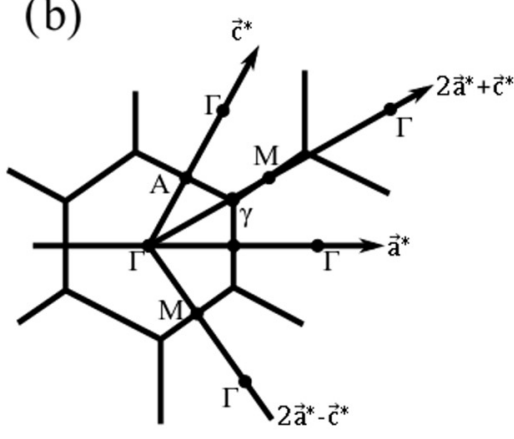

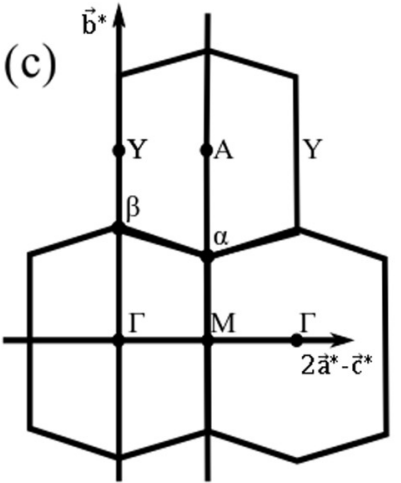

FIG. 13. (a) Dispersion of the low-lying phonon branches of the blue bronze measured between 225 and 295 K. The acoustic branches are labeled according to their polarization defined in the text. The hybridized phonon branches involved in the Peierls instability are colored in red and blue for the acoustic and optic counterparts, respectively. The empty green circles outline the phonon modes in the vicinity of the Kohn anomaly drawn at 230 K. (b) and (c): Sections of the Brillouin zone scanned during the inelastic scattering investigations (adapted from Refs. [21,76]).

(ii) for the shear deformation: $\mathrm{C}_{66}>\mathrm{C}_{44} \approx \mathrm{C}_{55}$, the shear deformation energy of the $(-201)$ layer of octahedra is stronger than that associated with their relative shift.

The dispersion of the phonon branch bearing the Kohn anomaly is not straightforward to analyze because it results from the hybridization of the acoustic mode polarized in the segment direction [label 2 and red lines in Fig. 13(a)] with a low lying optical phonon mode [blue lines in Fig. 13(a)] whose frequency is $1.4 \mathrm{THz}$ at the $\Gamma$ point and $1.8 \mathrm{THz}$ at the A point [blue circles in Fig. 13(a); the $\Gamma$ and A points are defined in Figs. 13(b) and 13(c)]. Although not measured from a $(2 \xi, 0,-\xi)$ scan along $\Gamma M$, the $\mathrm{TA}_{2}$ branch should be very close to the $\mathrm{TA}_{1}$ branch because $\mathrm{C}_{44} \approx \mathrm{C}_{55}$ (see Table I). At the zone boundary $\mathrm{M}$ point one expects a mixing of the $\mathrm{TA}_{2}$ branch with the above mentioned optical branch reaching $0.9 \mathrm{THz}$ at the $\mathrm{M}$ point along a different $(2 \xi, 0, \xi)$ scan [see Fig. 13(a)]. Another mixing between the two modes occurs near the A point, very close to the position of the Kohn anomaly. As a result, the low frequency phonon branch sustaining the Kohn anomaly should be strongly hybridized in the MA direction. The $(2 \xi, 0, \xi)$ scan clearly shows an avoided crossing near the $\Gamma$ point between the bare $\mathrm{LA}_{2}$ and the $1.4 \mathrm{THz}$ optical branches. Beyond this crossing point the frequency of the optical branch stays nearly constant until the $\gamma$ zone boundary crossing point. Then, the $\gamma \mathrm{M}$ scan shows that its frequency drastically decreases to reach $0.9 \mathrm{THz}$ at the $\mathrm{M}$ point (where it should mix with the $\mathrm{TA}_{2}$ branch). Such dispersion, which recalls that depicted in Fig. 12(c) cuts a preexisting valley of soft phonons discussed in the main text, where the frequency softening should be associated to correlated 1D Mo displacements, $\xi$. From the inverse of the half width of the wave vector softening along $\gamma \mathrm{M}$ it can be inferred a correlated Mo displacement on $\xi \approx 9 \AA$, which is about the distance between two neighboring clusters in the transverse layer direction [Fig. 2(a)]. The dispersion along MA is along the bottom of the valley of soft acoustic/optic phonons represented in Fig. 13(a). Note that this dispersion exhibits an extra screening with the 1D electron gas near $2 k_{F}$, which leads to the formation of a Kohn anomaly in the phonon spectrum. Finally, note the presence of four additional low-lying phonon modes at the position of the Kohn anomaly [empty green circles in Fig. 13(a)]. 
[1] C. Schlenker, J. Dumas, M. Greenblatt, and S. van Smaalen, eds., Physics and Chemistry of Low-Dimensional Inorganic Conductors, NATO ASI Series B, Physics Vol. 354 (Plenum, New York, 1996).

[2] J. Rouxel, ed., Crystal Chemistry and Properties of Materials with Quasi-One-Dimensional Structures (Reidel, Dordrecht, 1986).

[3] C. Schlenker, ed., Low Dimensional Electronic Properties of Molybdenum Bronzes and Oxides, Series: Physics and Chemistry of Materials with Low-Dimensional Structures (Springer, Netherlands, 1989).

[4] J. P. Pouget, in Physics and Chemistry of Low-Dimensional Inorganic Conductors, edited by C. Schlenker, J. Dumas, M. Greenblatt, and S. van Smaalen, NATO ASI Series B, Physics Vol. 354 (Plenum, New York, 1996), Chap. 11, pp. 185-217.

[5] S. Barisic, Phys. Rev. B 5, 932 (1972).

[6] S. Barisic, Phys. Rev. B 5, 941 (1972).

[7] J. Friedel, in Electron-Phonon Interactions and Phase Transitions, edited by T. Riste, NATO ASI B Vol. 29 (Plenum Press, New York, 1977), pp. 1-49.

[8] M. J. Rice and S. Strässler, Solid State Commun. 13, 125 (1973).

[9] J. Lindhard, Dan. Mat. Fys. Medd. 28, 1 (1954).

[10] W. L. McMillan, Phys. Rev. B 16, 643 (1977).

[11] J. P. Pouget, C. R. Phys. 17, 332 (2016).

[12] P. Y. Le Daeron and S. Aubry, J. Phys. Colloque C3 44, 1573 (1983).

[13] W. M. Lomer, Proc. Phys. Soc. 80, 489 (1962).

[14] M.-H. Whangbo and L. F. Schneemeyer, Inorg. Chem. 25, 2424 (1986).

[15] A. J. Berlinsky, Contemp. Phys. 17, 331 (1976).

[16] M. D. Johannes and I. I. Mazin, Phys. Rev. B 77, 165135 (2008).

[17] J. P. Pouget, C. Noguera, A. H. Moudden, and R. Moret, J. Phys. France 46, 1731 (1985)

[18] J. Graham and A. D. Wadsley, Acta Crystallogr. 20, 93 (1966).

[19] J. L. Mozos, P. Ordejón, and E. Canadell, Phys. Rev. B 65, 233105 (2002).

[20] G.-H. Gweon, J. W. Allen, R. Claessen, J. A. Clack, D. M. Poirier, P. J. Benning, C. G. Olson, W. P. Ellis, Y.-X. Zhang, L. F. Schneemeyer, J. Marcus, and C. Schlenker, J. Phys.: Condens. Matter 8, 9923 (1996).

[21] J. P. Pouget, S. Kagoshima, C. Schlenker, and J. Marcus, J. Physique Lett. 44, 113 (1983).

[22] G. Travaglini, P. Wachter, J. Marcus, and C. Schlenker, Solid State Commun. 37, 599 (1981).

[23] J. P. Pouget, C. Escribe-Filipini, B. Hennion, R. Currat, A. HMoudden, R. Moret, J. Marcus, and C. Schlenker, Mol. Cryst. Liq. Cryst. 121, 111 (1985).

[24] J. P. Pouget, B. Hennion, C. Escribe-Filippini, and M. Sato, Phys. Rev. B 43, 8421 (1991).

[25] P. Hohenberg and W. Kohn, Phys. Rev. 136, B864 (1964).

[26] W. Kohn and L. J. Sham, Phys. Rev. 140, A1133 (1965).

[27] J. M. Soler, E. Artacho, J. D. Gale, A. García, J. Junquera, P. Ordejón, and D. Sánchez-Portal, J. Phys.: Condens. Matter 14, 2745 (2002).

[28] E. Artacho, E. Anglada, O. Diéguez, J. D. Gale, A. García, J. Junquera, R. M. Martin, P. Ordejón, J. M. Pruneda, D. SánchezPortal, and J. M. Soler, J. Phys.: Condens. Matter 20, 064208 (2008).
[29] J. P. Perdew, K. Burke, and M. Ernzerhof, Phys. Rev. Lett. 77, 3865 (1996).

[30] N. Troullier and J. L. Martins, Phys. Rev. B 43, 1993 (1991).

[31] L. Kleinman and D. M. Bylander, Phys. Rev. Lett. 48, 1425 (1982).

[32] S. G. Louie, S. Froyen, and M. L. Cohen, Phys. Rev. B 26, 1738 (1982).

[33] E. Artacho, D. Sànchez-Portal, P. Ordejón, A. García, and J. M. Soler, Phys. Status Solidi B 215, 809 (1999).

[34] H. J. Monkhorst and J. D. Pack, Phys. Rev. B 13, 5188 (1976).

[35] M. Ghedira, J. Chenavas, M. Marezio, and J. Marcus, J. Solid State Chem. 57, 300 (1985).

[36] E. Canadell and M.-H. Whangbo, Chem. Rev. 91, 965 (1991).

[37] U. V. Waghmare, H. Kim, I. J. Park, N. Modine, P. Maragakis, and E. Kaxiras, Comput. Phys. Commun. 137, 341 (2001).

[38] A. V. Fedorov, S. A. Brazovskii, V. N. Muthukumar, P. D. Johnson, J. Xue, L.-C. Duda, K. E. Smith, W. H. McCarroll, M. Greenblatt, and S. L. Hulbert, J. Phys.: Condens. Matter 12, L191 (2000).

[39] K. E. Smith, J. Xue, L. Duda, A. Fedorov, P. D. Johnson, S. L. Hulbert, W. McCarroll, and M. Greenblattd, J. Electron Spectrosc. Relat. Phenom. 117-118, 517 (2001).

[40] G.-H. Gweon, J. D. Denlinger, J. W. Allen, R. Claessen, C. G. Olson, H. Höchst, J. Marcus, C. Schlenker, and L. F. Schneemeyer, J. Electron Spectrosc. Relat. Phenom. 117-118, 481 (2001).

[41] L. Perfetti, S. Mitrovic, G. Margaritondo, M. Grioni, L. Forró, L. Degiorgi, and H. Höchst, Phys. Rev. B 66, 075107 (2002).

[42] H. Ando, T. Yokoya, K. Ishizaka, S. Tsuda, T. Kiss, S. Shin, T. Eguchi, M. Nohara, and H. Takagi, J. Phys.: Condens. Matter 17, 4935 (2005).

[43] M. Grioni, S. Pons, and E. Frantzeskakis, J. Phys.: Condens. Matter 21, 023201 (2009).

[44] H. Y. Liu, I. Gierz, J. C. Petersen, S. Kaiser, A. Simoncig, A. L. Cavalieri, C. Cacho, I. C. E. Turcu, E. Springate, F. Frassetto, L. Poletto, S. S. Dhesi, Z.-A. Xu, T. Cuk, R. Merlin, and A. Cavalleri, Phys. Rev. B 88, 045104 (2013).

[45] D. Mou, R. M. Konik, A. M. Tsvelik, I. Zaliznyak, and X. Zhou, Phys. Rev. B 89, 201116(R) (2014).

[46] W. J. Schutte and J. L. D. Boer, Acta Crystallogr. B 49, 579 (1988).

[47] E. Machado-Charry, P. Ordejón, E. Canadell, C. Brun, and Z. Z. Wang, Phys. Rev. B 74, 155123 (2006).

[48] C. Noguera and J.-P. Pouget, J. Phys. I France 1, 1035 (1991).

[49] D. Allender, J. W. Bray, and J. Bardeen, Phys. Rev. B 9, 119 (1974).

[50] G. A. Toombs, Phys. Rep. 40, 181 (1978).

[51] S. Girault, A. H. Moudden, and J. P. Pouget, Phys. Rev. B 39, 4430 (1989).

[52] J.-P. Pouget, P. Alemany, and E. Canadell, Materials Horizons 5, 590 (2018).

[53] P. A. Lee, T. M. Rice, and P. W. Anderson, Phys. Rev. Lett. 31, 462 (1973).

[54] H. J. Schultz, in Low-Dimensional Conductors and Superconductors, edited by D. Jérome and L. G. Caron, NATO ASI B155 (Plenum Press, New York, 1987), p. 95.

[55] G. Travaglini, I. Morke, and P. Wachter, Solid State Commun. 45, 289 (1983).

[56] R. Comès and G. Shirane, Phys. Rev. B 5, 1886 (1972). 
[57] R. Currat, R. Comès, B. Dorner, and E. Wiesendanger, J. Phys. C: Solid State Phys. 7, 2521 (1974).

[58] R. Currat, H. Buhay, C. H. Perry, and A. M. Quittet, Phys. Rev. B 40, 10741 (1989).

[59] J.-P. Pouget, S. M. Shapiro, and K. Nassau, J. Phys. Chem. Solids 40, 267 (1979).

[60] A. Ottolenghi and J.-P. Pouget, J. Phys. I France 6, 1059 (1996).

[61] P. Foury-Leylekian and J.-P. Pouget, Solid State Sci. 4, 387 (2002).

[62] B. Hennion, J.-P. Pouget, and M. Sato, Phys. Rev. Lett. 68, 2374 (1992).

[63] S. Ravy, H. Requardt, D. Le Bolloc'h, P. Foury-Leylekian, J.-P. Pouget, R. Currat, P. Monceau, and M. Krisch, Phys. Rev. B 69, 115113 (2004).

[64] P. Monceau, Adv. Phys. 61, 325 (2012).

[65] B. Guster, M. Pruneda, P. Ordejón, E. Canadell, and J.-P. Pouget (unpublished).

[66] H. Requardt, J. E. Lorenzo, P. Monceau, R. Currat, and M. Krisch, Phys. Rev. B 66, 214303 (2002).

[67] S. van Smaalen, J. L. de Boer, A. Meetsma, H. Graafsma, H.-S. Sheu, A. Darovskikh, P. Coppens, and F. Levy, Phys. Rev. B 45, 3103 (1992).

[68] J. P. Pouget, R. Moret, A. Meerschaut, L. Guemas, and J. Rouxel, J. Phys. Colloque C3 44, 1729 (1983).
[69] M. Maschek, S. Rosenkranz, R. Heid, A. H. Said, P. GiraldoGallo, I. R. Fisher, and M. Weber, Phys. Rev. B 91, 235146 (2015).

[70] K. Rossnagel, J. Phys.: Condens. Matter 23, 213001 (2011).

[71] F. Weber, S. Rosenkranz, J.-P. Castellan, R. Osborn, R. Hott, R. Heid, K.-P. Bohnen, T. Egami, A. H. Said, and D. Reznik, Phys. Rev. Lett. 107, 107403 (2011).

[72] E. Canadell and M.-H. Whangbo, Phys. Rev. B 43, 1894 (1991).

[73] E. Sandre, P. Foury-Leylekian, S. Ravy, and J. P. Pouget, Phys. Rev. Lett. 86, 5100 (2001).

[74] B. Guster, D. Boskovic, M. Pruneda, P. Ordejón, E. Canadell, and J.-P. Pouget (unpublished).

[75] P. Foury and J. P. Pouget, Int. J. Mod. Phys. B 7, 3973 (1993).

[76] C. Escribe-Filipini, J.-P. Pouget, R. Currat, B. Hennion, and J. Marcus, in Charge Density Waves in Solids, edited by G. Hutiray and Sólyom, Lecture Notes in Physics Vol. 217 (SpringerVerlag, Berlin, 1985), pp. 71-75.

[77] J. W. Brill, in Handbook of Elastic Properties of Solids, Liquids and Gases, Vol. II: Elastic Properties of Solids; Theory, Elements and Compounds, Novel Materials, Alloys and Building Materials, edited by M. Levy, H. Bass, and R. Stern (Academic Press, New York, 2001), Chap. 10, pp. 143-162. 